\title{
Influence of Machining Environment on Surface Integrity in HSM of Inconel 718 with Productivity Perspective
}

\author{
G. $\operatorname{Kadam}^{1 *}$, R. Pawade ${ }^{2}$ \\ ${ }^{1}$ Assistant Professor, SIES GST, Nerul, Navi Mumbai, India \\ ${ }^{2}$ Associate Professor, DBATU, Lonere, Raigad,India \\ \{ganeshkadam2020@yahoo.com\}
}

\begin{abstract}
This paper investigates the effect of material removal rate (MRR) and mainly machining environment on resultant surface integrity in high-speed machining (HSM) of Inconel 718. For this high-speed turning is carried out for three different cutting speeds, feedrates and depth of cut under dry, flood coolant and water vapour machining environments. The results show that flood cooling is ineffective in high-speed machining. Water vapours acts as a coolant and lubricant, and hence is highly effective in lowering surface roughness and surface damages in machining. However at very high material removal rates, dry machining seems to be an economical option. Under all the environments the residual stresses were found to be compressive in nature at all MRR levels. Keeping in view green manufacturing, good productivity and better quality, it is suggested that machining of Inconel 718 under water vapour environment is an ideal approach.
\end{abstract}

Keywords: High speed machining, Inconel 718, water vapour, surface integrity, green manufacturing

\section{Introduction}

Superalloy, Inconel 718 , is the most commonly employed nickel-based alloy and it finds wide applications majority being in aerospace. But it is also stamped under most difficult-to-cut materials mainly because of the poor machinability properties which are [1-4]: major strength retained during machining, high strain rate sensitive nature, readily work hardening characteristics, highly abrasive carbide particles in microstructure and poor thermal conductivity leading to high cutting temperatures. Attempts have been made in the past with due focus on surface integrity induced by turning operation of Inconel 718 considering the machining environment. The reported findings state reduction in surface roughness under different machining environments like wet [5, 6], MQL [7, 8], hybrid machining [9], cooling air and minimum quantity lubrication (CAMQL) [10] and liquid nitrogen (LN2) [11]. Further the beneficial effects of machining environment in inducing lesser tensile or more compressive residual stresses have been also reported for wet [5,6] and cryogenic [12] environments, to mention a few. Podgorkov and Godlevski around 1990s proposed a new pollution-free cutting technique with water vapour as coolant and lubricant in machining, and further works on it reported decrease in surface roughness of various work materials like C45 steel [13], ANSI 304 stainless steel [14] and Titanium alloy (Ti$6 \mathrm{Al}-4 \mathrm{~V})$ [15]. Thus keeping a keen eco-friendly perspective, the present work focuses to explore surface integrity aspects in turning of Inconel 718 with due addressing to productivity.

\section{Experimental Work}

Considering the present competitive manufacturing demands of higher production, the machining parameters were wisely selected confining high-speed regime to achieve significantly higher MRR as in Fig. 1a. Further, it was proposed to carry out the experiments under three different environments - dry, flood coolant and water vapour. The performance indicators selected for analysing the surface integrity were surface roughness, surface damage and the surface residual stresses [16]. Fully Annealed Inconel 718 ring specimens with OD $58 \mathrm{~mm}$, ID $46 \mathrm{~mm}$ and wall thickness of $5 \mathrm{~mm}$ were used as a work material by fitting it over special mandrel (see Fig. 1b and c).

B. Iyer, S. Nalbalwar and R. Pawade (Eds.)

ICCASP/ICMMD-2016. Advances in Intelligent Systems Research.

Vol. 137, Pp. 89-93.

(c) 2017. The authors - Published by Atlantis Press

This is an open access article under the CC BY-NC license (http://creativecommons.org/licens)es/by-nc/4.0/). 
The chemical composition of Inconel 718 was Ni 54.95, Cr 17.90, Fe 16.54, Nb 4.85, Ti 0.92, Co 0.92, Al 0.52, Si 0.08 and C 0.03. CNMG120408MS PVD-TiAlN coated tungsten carbide inserts of grade KC5510 (Make Kernametal) along with PCLNR2525MS tool holder (Make WIDIA) were used as cutting tool. The insert are rhomboid shaped, have included angle of $80^{\circ}$, nose radius of $0.8 \mathrm{~mm}$, a positive rake angle of $13^{\circ}$, and $95^{\circ}$ approach angle after mounting in tool holder $[17,18]$. The turning experiments were performed on CNC lathe (Make Micromatic Ace, Jobber XL). For experiments under water vapour as a coolant/lubricant environment, assistance of steam generation device (Fig. 1d) was taken with temperature and pressure of the steam maintained at around $105{ }^{\circ} \mathrm{C}$ and 1.6 bar respectively. Convergent type nozzles were used to eject the water vapour on the cutting zone. Fresh cutting tip was used for every experiment. After the experiments, the measurement of surface roughness, surface damage and circumferential (hoop) residual stresses was done using surface roughness tester Mitutoyo SJ-301, Scanning Electron Microscopy (SEM) ZEISS Ultra FESEM and Philips Panalytical microarea residual stress analyser $\left(\sin ^{2} \emptyset\right.$ technique) respectively. All the experiments were repeated and re-measurements were done to validate the data.
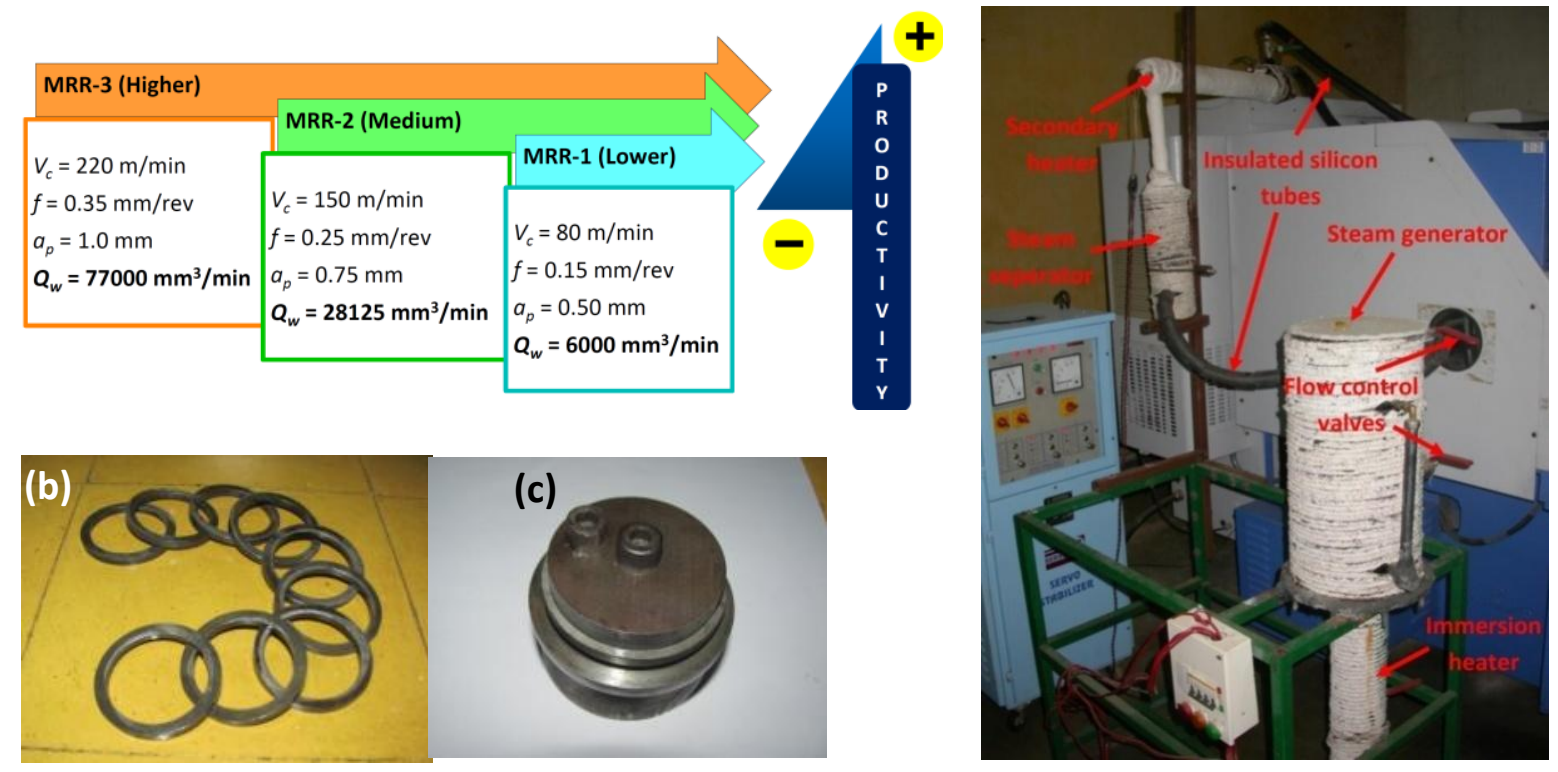

Fig. 1. (a) Selected machining parameters with MRR and productivity levels, (b) Workpieces - Inconel 718 rings, (c) Mandrel with mounted Inconel 718 ring and, (d)Steam generation device.

\section{Results and discussion}

\subsection{Analysis of surface roughness}

Fig. 2a shows that increase in MRR cause an increase in surface roughness which is quite obvious as per fundamentals of machining. However, at a given MRR, the surface roughness varies according to different machining environment which justifies its importance. At low MRR, it is seen that the surface roughness is lowest for water vapor environment as it acts as lubricant and coolant, and hence mainly because of its better lubricating action as well as excellent penetration ability which is in well agreement with [13-15]. In case of dry cutting, sufficiently high temperatures are created which cause thermal softening leading to restructuring of the machined surface layer as well as reduced stresses on tool assisting in ease of material removal, and thus lower surface roughness value is seen as compared to flood coolant environment. However the surface roughness is highest in case of flood coolant environment, as the lubrication and cooling action of flood coolant prevents temperature rise and reduces thermal softening imparting stresses on cutting tool deteriorating the surface finish.Further at medium MRR, the surface roughness value in case of flood coolant environment is again highest, but that under dry and water vapor environment is almost the same. In case of flood coolant environment, the lubrication action of fluid is reduced due to very limited access of the fluid to the toolworkpiece or tool-chip interfaces and only cooling action dominates leading to deterioration in surface finish. For the case of dry cutting, increased MRR causes higher cutting temperatures and hence the high degree of thermal softening assists for easy material removal leading to lower surface roughness.Further for water vapor environment, increase in MRR degrades the penetration performance of water vapor and also the storing time is reduced. As a result water vapor is not able to form an effective low shearing strength lubricant layer and hence surface roughness increases. 

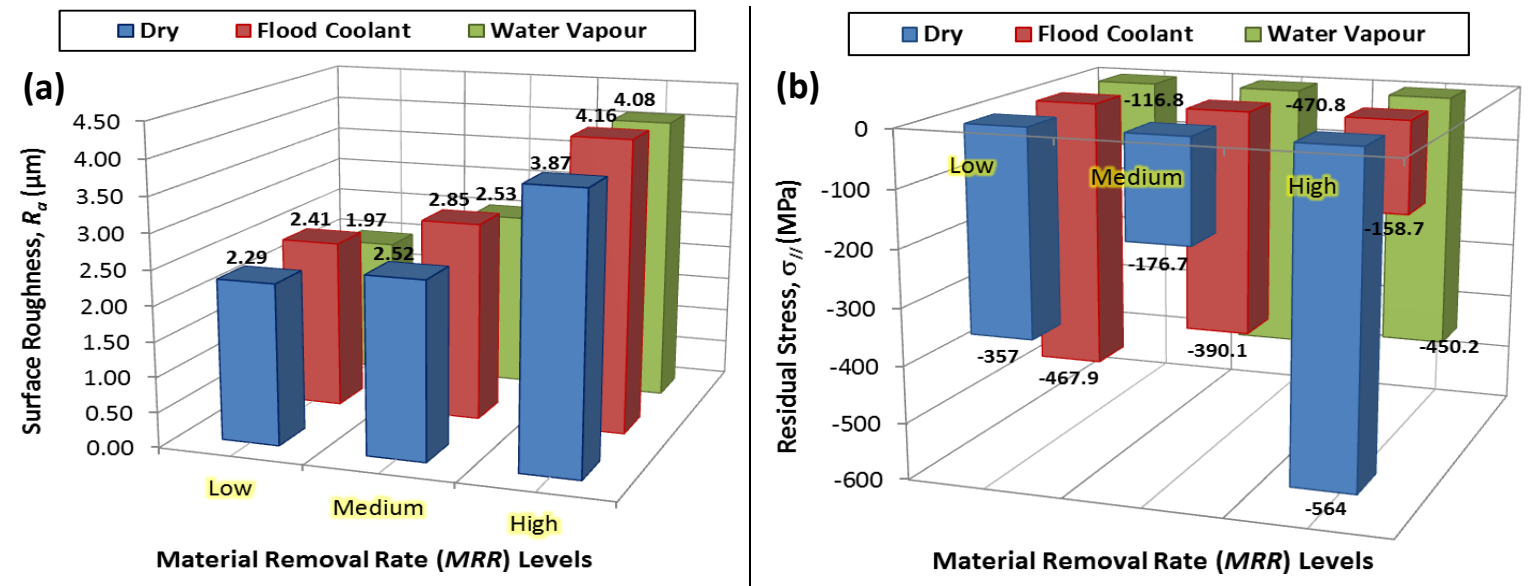

Fig. 2. Effect of MRR on (a) Surface roughness and (b) Hoop residual stresses under different environments; Low MRR: $6000 \mathrm{~mm}^{3} / \mathrm{min}$; Medium MRR: $28125 \mathrm{~mm}^{3} / \mathrm{min}$; High MRR: $77000 \mathrm{~mm}^{3} / \mathrm{min}$

Drifting finally to the highest MRR level, the surface roughness values in case of flood coolant again comparatively remains at the peak of all. This is because the penetration capability of fluid to the tool-workpiece or tool-chip interface ceases at such very high-speed conditions. Further high temperatures are generated, due to which the fluid tends to be vaporized forming a high temperature blanket that reduces cooling efficiency thus leading to higher surface roughness. Considering the case of dry cutting, the increased temperatures do assist in easy material removal partly due to softening effect; but a characteristic noise was also generated which emphasizes that the machining was under heavy ploughed compression and also there was slight burnishing effect. Hence this combined effect leads to lower surface roughness values. For water vapor environment, the surface roughness is found to be higher than dry conditions mainly because the effectiveness of water vapor degrades severely at this highest MRR level. The penetration capability of water vapor drastically reduces and the storing time also reduces leading to increased surface roughness. It is thus understood that at the low MRR and medium MRR level, the water vapor environment is quite effective for achieving better surface finish; however dry environment seems to be a better option at the high MRR level.

\subsection{Analysis of surface damage}

The micrographs of examined machined surfaces at magnifications from 100x-1000x through SEM revealed material side flow, grooves, microparticle deposits, smeared material and white layers as the characteristic surface alterations (Fig. 3). The machining environment and MRR, both influenced the occurrences and magnitude of the defects. Overall, the lowest MRR level under water vapor environment comparatively showed the least quantity of material side flow than dry and flood coolant environment. Shallow grooves along the feed spacing were seen in all cases and their quantity and severity increased with MRR. The grooves were found to be severe in dry machining, while almost light to moderate in case of flood coolant and water vapor environment which can be mainly attributed to their cooling and lubricating effect. Microparticle deposits typically were almost negligible in flood cooling only which was mainly because of flushing action of cutting fluid. Smeared material of around $47.175 \mu \mathrm{m}$ width was mainly visible at the highest MRR level in case ofwater vapor environment only. The white layers were the most severe in case of dry machining as compared to other environments.
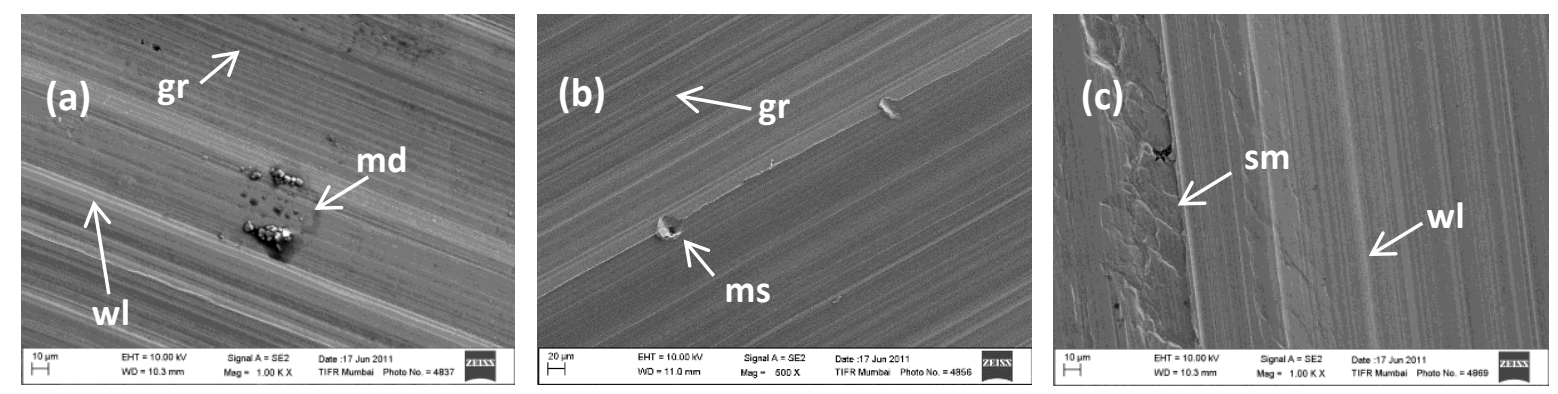

Fig. 3. (a) Dry, medium MRR (28125 mm3/min): 1000x, (b) Flood coolant, high MRR (77000 mm3/min): 500x, (c) Water Vapor, high MRR (77000 mm3/min): 1000x;gr - Shallow grooves, md Microparticle deposits, ms - Material sideflow, sm - Smeared material, wl - White layers 


\subsection{Analysis of residual stresses}

From Fig. 2b, it is learnt that an increase in MRR causes changes in residual stresses and they further vary drastically under different machining environments. The resulting stress state after machining is a combination of thermal and mechanical effects. It is known that the thermally dominant machining deformation leaves behind tensile residual stress in the machined surface, whereas mechanically dominant machining deformation induces compressive residual stress $[1,3]$.

It is seen that in dry machining, at the lowest MRR, the strain rate and hence heat generated in the shear zone are comparatively the lowest, due to which mechanical loading effect dominates than the thermal effect leading to compressive residual stresses. However at medium MRR level, the heat generated in the shear zone increases due to increase in strain rate and the thermal effects dominate shifting the residual stress towards tensile direction.

Further at the highest MRR level, the residual stress shifts downwards and achieves the highest compressive level. This can be attributed to the fact that high MRR rate causes higher chip flow rates due to increased cutting speed thus reducing the length of time available for the heat generated in shear zone to diffuse into the workpiece surface and consequently increasing the amount of thermal energy evacuated in the chip. This reduces the effect of the thermal load on the workpiece and therefore mechanical effects dominate, resulting into more compressive residual stress regime.

In case of flood coolant environment, with increase in MRR the residual stresses shift towards tensile direction, however remain well within compressive region. At low MRR the mechanical effects dominate leading to higher compressive residual stress as the strain rate is low leading to less shearing heat generation, and also due to low cutting temperatures due to the quite effective lubricating and cooling action of the flood coolant. With increase to medium MRR level, the thermal load on the workpiece increases and comparatively less compressive residual stress value is seen which can be hence attributed to increased shearing and strain rate causing higher hear generation and also to further lubricating and cooling action of flood coolant being not sufficiently effective at such increased cutting speeds leading to higher temperatures. Further at highest MRR level, the residual stress shifts towards the tensile direction due to the appreciably increased thermal load on the workpiece leading to dominance of thermal effects, which is also mainly due to the flood coolant vapor blanket formation drastically reducing the heat transfer rate along with effects of very high shearing and strain rate.

In case of water vapor environment increment in MRR leads to residual stress attaining higher compressive values. At the lowest MRR level, the thermal effects dominate more than the mechanical effect as heat transfer prevails from chips to the workpiece during their longer stay in machining zone and hence less compressive residual stress value near tensile regime is encountered. However at medium MRR level, the residual stress moves in compressive direction and attains higher value mainly due to dominance of mechanical effects as majority of heat being flow away by the thicker chips bulk thus relieving workpiece thermal load. Further athighest MRR level, there is almost negligible change in residual stress value which is mainly due to dominance of mechanical effects as in earlier case.

\section{Conclusions}

Overall, it can be concluded that both, MRR and machining environment, significantly influence the surface finish, surface alterations and machining induced residual stress to variable extents in high-speed turning of Inconel 718. Water vapour as a coolant and lubricant in machining is effective only at lower MRR and its effect gets degraded at higher MRR due to poor penetrability to the tool-work and tool-chip interface at higher cutting speeds. Flood cooling tends to be ineffective as MRR is increased and it cannot perform the action of cooling and lubrication satisfactorily in high-speed machining range to an appreciable extent. The residual stresses are compressive in nature under all the MRR levels and under different machining environments. Thus a better surface integrity in high-speed machining can be obtained at lower MRR under water vapour environment while at medium and mainly at higher MRR dry machining seems to be the best choice.

\section{Acknowledgement}

Authors would like to thank Santosh Utekar (United Metal Industries Bombay Pvt. Ltd., Mumbai), Bhagyashree Chalke (TIFR, Colaba, Mumbai) and OIM \& Texture lab (IIT Bombay, Powai) for assistance in heat treatment 
of Inconel 718 rings, scanning electron microscopy of machined samples and XRD residual stress measurements respectively.

\section{References}

[1]. R.S. Pawade, Suhas S. Joshi, P.K. Brahmankar, Effect of machining parameters and cutting edge geometry on surface integrity of high-speed turned Inconel 718, International Journal of Machine Tools \& Manufacture 48 (2008) 15-28.

[2]. C. Courbon, D. Kramar, P. Krajnik, F. Pusavec, J. Rech, J. Kopac, Investigation of machining performance in high-pressure jet assisted turning of Inconel 718: An experimental study, International Journal of Machine Tools \& Manufacture 49 (2009) 1114-1125.

[3]. D. Dudzinski, A. Devillez, A. Moufki, D. Larrouquère, V. Zerrouki, J. Vigneau, A review of developments towards dry and high speed machining of Inconel 718 alloy, International Journal of Machine Tools \& Manufacture 44 (2004) 439-456.

[4]. R.S. Pawade, Suhas S. Joshi, P.K. Brahmankar, M. Rahman, An investigation of cutting forces and surface damage in high-speed turning of Inconel 718, Journal of Materials Processing Technology 192193 (2007) 139-146.

[5]. R.M. Arunachalam, M.A. Mannan, A.C. Spowage, Residual stress and surface roughness when facing age hardened Inconel 718 with $\mathrm{CBN}$ and ceramic cutting tools, International Journal of Machine Tools \& Manufacture 44 (2004) 879-887.

[6]. R.M. Arunachalam, M.A. Mannan, A.C. Spowage, Surface integrity when machining age hardened Inconel 718 with coated carbide cutting tools, International Journal of Machine Tools \& Manufacture 44 (2004) 1481-1491.

[7]. Y. Kamata, T. Obikawa, High speed MQL finish-turning of Inconel 718 with different coated tools, Journal of Materials Processing Technology 192-193 (2007) 281-286.

[8]. Toshiyuki Obikawa, Yasuhiro Kamata, Yuki Asano, Kousuke Nakayama, Andrew W. Otieno, Microliter lubrication machining of Inconel 718, International Journal of Machine Tools \& Manufacture 48 (2008) 1605-1612.

[9]. Z.Y. Wang, K.P. Rajurkar, J. Fan, S. Lei, Y.C. Shin, G. Petrescu, Hybrid machining of Inconel 718, International Journal of Machine Tools \& Manufacture 43 (2003) 1391-1396.

[10]. Y. Su, N. He, L. Li, A. Iqbal, M.H. Xiao, S. Xu, B.G. Qiu, Refrigerated cooling air cutting of difficultto-cut materials, International Journal of Machine Tools \& Manufacture 47 (2007) 927-933.

[11]. Z.Y. Wang, K.P. Rajurkar, Cryogenic machining of hard-to-cut materials, Wear 239 (2000) 168-175.

[12]. F. Pusavec, H. Hamdi, J. Kopac, I.S. Jawahir, Surface integrity in cryogenic machining of nickel based alloy - Inconel 718, Journal of Materials Processing Technology 211 (2011) 773-783.

[13]. Junyan Liu, Rongdi Han, Yongfeng Sun, Research on experiments and action mechanism with water vapor as coolant and lubricant in Green cutting, International Journal of Machine Tools \& Manufacture 45 (2005) 687-694.

[14]. Liu Junyan, Liu Huanpeng, Han Rongdi, Wang Yang, The study on lubrication action with water vapor as coolant and lubricant in ANSI 304 stainless steel, International Journal of Machine Tools \& Manufacture 50 (2010) 260-269.

[15]. Raju S. Pawade, D.S.N. Reddy, Ganesh S. Kadam, Chip segmentation behaviour and surface topography in high-speed turning of titanium alloy (Ti-6Al-4V) with eco-friendly water vapour, International Journal of Machining and Machinability of Materials 13-2/3 (2013) 113-137.

[16]. Ganesh S. Kadam, Analysis and modelling of the effect of machining environment on surface integrity in high speed turning of Inconel 718, M.Tech. Thesis Report, 2010-2011, DBATU, Lonere, Raigad, M.S., India.

[17]. Turning Tooling Catalog 8010, Kennametal, pp. 2-41.

[18]. Widia Turning 2010 Catalog, Widia, pp. A2-A35. 